

\title{
TBK1 at the crossroad of signalling pathways
}

\section{$(96$}

TBK 1 is

responsible

for crossover

between

inflammation

and energy

expenditure
TANK-binding kinase 1 (TBK1) is known to be activated by pro-inflammatory cytokines, but its role in metabolism has been unclear. New research, led by Peng Zhao, now shows that TBK1 suppresses inflammation and energy expenditure in adipose tissue via two separate pathways, indicating that TBK1 is positioned at a crossroad between energy sensing and inflammatory signalling pathways.

Previous work showed that I $\mathrm{B}$ kinase- $\varepsilon$ (IKKe) was increased in obesity and suppressed energy expenditure, leading to the discovery of amlexanox, which inhibits IKKe and TBK1. As a result of this work, it was thought that IKKe and TBK1 had similar effects; however, this turned out not to be the case. "After we did the adipocyte-specific knockout of TBK1, it became obvious that they had different roles," explains corresponding author Alan Saltiel. "We now think that IKKe represses cAMP signalling, while TBK1 represses AMPK; these are the two major mechanisms of energy expenditure in fat."

In an adipocyte-specific TBK1knockout (ATKO) mouse model, the authors demonstrated that high-fatdiet-induced obesity was attenuated via an increase in energy expenditure. In addition, TBK1 was shown to inhibit AMPK, resulting in reduced respiration and increased energy storage. In turn, AMPK activated TBK1, which could explain how TBK1 limits the stimulation of energy expenditure during caloric restriction.

The ATKO model also displayed increased adipose tissue inflammation and insulin resistance. The researchers showed that TBK1 suppresses inflammation by phosphorylating and inducing the degradation of nuclear factor- $\kappa \mathrm{B}$ (NF- $\kappa \mathrm{B}$ )-inducing kinase (NIK), which is upstream of NF- $\kappa B$. In adipocytes, NF- $\mathrm{KB}$ induced the activity of TBK1, providing a possible explanation for how inflammation in adipocytes is self-limited. "The clincher of the paper is that because of its placement in these two feedback loops, TBK1 is responsible for crossover between inflammation and energy expenditure, which explains how obesity-induced inflammation represses AMPK (and energy expenditure), and how caloric restriction (through activation of AMPK) represses NF- $\mathrm{B}$ (and inflammation)," comments Saltiel.

"These data suggest that TBK1 inhibitors might act synergistically with AMPK activators, to take the brakes off energy expenditure," explains Saltiel. "We are planning both preclinical and clinical studies using our TBK1 inhibitor amlexanox together with drugs like metformin; it will be interesting to see what happens." More work needs to be done to further determine the role of other factors in these pathways, as well as the function of the pathways in tissues affected by obesity. "I think that it is interesting how deep exploration of signalling pathways leads us to new ideas for how to use [the experimental drug amlexanox]," concludes Saltiel.

Claire Greenhill

ORIGINAL ARTICLE Zhao, P. et al. TBK1 at the crossroads of inflammation and energy homeostasis in adipose tissue. Cell 172, 731-743 (2018) FURTHER READING Reilly, S. M. \& Saltiel, A. R. Adapting to obesity with adipose tissue inflammation. Nat. Rev. Endocrinol. 13, 633-643 (2017) 